Copyright (C) 2005 IEEE. Reprinted from

IEEE Transactions on Biomedical Engineering, 2005; 52 (12):2112-2115

This material is posted here with permission of the IEEE. Such permission of the IEEE does not in any way imply IEEE endorsement of any of the University of Adelaide's products or services. Internal or personal use of this material is permitted. However, permission to reprint/republish this material for advertising or promotional purposes or for creating new collective works for resale or redistribution must be obtained from the IEEE by writing to pubs-permissions@ieee.org.

By choosing to view this document, you agree to all provisions of the copyright laws protecting it. 


\section{Short- and Long-Term Joint Symbolic Dynamics of Heart Rate and Blood Pressure in Dilated Cardiomyopathy}

Mathias Baumert, Vico Baier, Sandra Truebner, Alexander Schirdewan, and Andreas Voss*

\begin{abstract}
Autonomic cardiovascular control involves complex interactions of heart rate and blood pressure. In patients with dilated cardiomyopathy (DCM), this control is impaired and parameters for its quantification might be of prognostic importance. In this paper, we introduce methods based on joint symbolic dynamics (JSD) for the enhanced analysis of heart rate and blood pressure interactions. To assess the coarse-grained dynamics beat-to-beat changes of heart rate and blood pressure are encoded in symbol strings. Subsequently, the distribution properties of short symbol sequences (words) as well as the scaling properties of the whole symbol string are assessed. The comparison of joint symbolic heart rate and blood pressure dynamics in DCM $(n=75)$ with those in healthy controls $(n=75)$ showed significant changes. Both, the distribution of words and the scaling properties indicate a loss in heart rate dynamics associated with blood pressure regulation in DCM. In conclusion, the analyses of short- and long-term JSDs provide insights into complex physiological heart rate and blood pressure interactions and furthermore reveal patho-physiological cardiovascular control in DCM.
\end{abstract}

Index Terms-Blood pressure variability, dilated cardiomyopathy, heart rate variability, symbolic dynamics.

\section{INTRODUCTION}

The analysis of heart rate variability (HRV) provides clinically relevant insights into autonomic control [1]. Short-term HRV is strongly connected with blood pressure regulation through the baroreflex (i.e. the compensation of rapid blood pressure changes via heart rate adjustment) [2]. Consequently, only the bivariate analysis of heart rate and blood pressure variability can provide insights into such mechanisms of cardiovascular control. Several standard signal processing tools, such as cross-correlation [3], cross-spectral analysis [4] or model based approaches [5] were applied. However, the mostly used approach to estimate the baroreflex sensitivity is the sequence method [6] (see Methods section).

Since the relationship between heart rate and blood pressure involves complex interactions, they cannot be sufficiently described by linear models [7]. Some of the already introduced bivariate nonlinear approaches include mutual information [8] and conditional entropy [9]. In this paper, we present a new methodology for heart rate and blood pressure interaction analysis based on their short- and long-term joint symbolic dynamics (JSD). The concept of symbolic dynamics goes back to J. S. Hadamard (1898) [10] and allows a simplified description of the dynamics of a system with a limited amount of symbols. Methods based on symbolic dynamics have already been successfully applied to HRV analysis providing some more global information about the underlying system [11], [12].

Manuscript received July 8, 2004; revised April 17, 2005. This work was supported in part by the Deutsche Forschungsgemeinschaft under Grant DFG Vo505/3-1 and Grant DFG Vo505/4-2 and in part by the German Federal Ministry of Economics and Labor under Grant BMWA-PRO INNO, KF 0317302KLF3. Asterisk indicates corresponding author.

M. Baumert, V. Baier, and S. Truebnera are with the Department of Medical Engineering, University of Applied Sciences Jena, 07745 Jena, Germany.

A. Schirdewan is with the Humboldt-University Berlin, Charitè, FranzVolhard-Hospital, 13125 Berlin, Germany.

*A. Voss is with the Department of Medical Engineering, Carl-ZeissPromenade 2, University of Applied Sciences Jena, 07745 Jena, Germany (e-mail: voss@fh-jena.de).

Digital Object Identifier 10.1109/TBME.2005.857636
The introduced methodology was applied to investigate patients with dilated cardiomyopathy (DCM) who are characterized by a dilated myocardium, an impaired myocardial performance and a high mortality rate. DCM leads to a reduced HRV as well as a reduced baroreflex sensitivity [13].

\section{METHODS}

\section{A. Patients and Data Preprocessing}

Heart rate and blood pressure data of 75 patients with DCM and 75 healthy controls $(\mathrm{CON})$ with comparable age $(\mathrm{CON}: 48 \pm 10$ years versus DCM: $50 \pm 8$ years) were analyzed. All patients had a stable sinus rhythm during recording and were under medical treatment. Ectopic beats of 0.09 [0-0.46] \% occurred in DCM patients (median [interquartil range]) whereas a total of 57 ectopic beats occurred in 12 controls. High-resolution ECG and continuous blood pressure (Portapres M2 monitor, volume clamp method) were recorded under standardized resting conditions in supine position over $30 \mathrm{~min}$. Time series of beat-to-beat intervals (BBI) and systolic blood pressure values (SP) were extracted automatically and, thereafter, visually inspected. Ectopic beats were determined, removed and interpolated using an algorithm based on local variance estimation [14].

\section{B. Joint Symbolic Dynamics}

In $\mathbf{X}(1), x^{\mathrm{BBI}}$ and $x^{\mathrm{SP}}$ are $n$ beat-to-beat values of BBI and SP, respectively

$$
X=\left\{\left[x_{n}^{\mathrm{BBI}}, x_{n}^{\mathrm{SP}}\right]^{T}\right\}_{n=0,1, \ldots} x \in R
$$

$\mathbf{X}$ is transformed in $\mathbf{S}(2)$ defined as

$$
S=\left\{\left[s_{n}^{\mathrm{BBI}}, s_{n}^{\mathrm{SP}}\right]^{T}\right\}_{n=0,1, \ldots} s \in 0,1
$$

with the following definitions:

$$
\begin{gathered}
s_{n}^{\mathrm{BBI}}=\left\{\begin{array}{l}
0:\left(x_{n}^{\mathrm{BBI}}-x_{n+1}^{\mathrm{BBI}}\right) \leq l^{\mathrm{BBI}} \\
1:\left(x_{n}^{\mathrm{BBI}}-x_{n+1}^{\mathrm{BBI}}\right)>l^{\mathrm{BBI}}
\end{array}\right. \\
s_{n}^{\mathrm{SP}}=\left\{\begin{array}{l}
0:\left(x_{n}^{\mathrm{SP}}-x_{n+1}^{\mathrm{SP}}\right) \leq l^{\mathrm{SP}} \\
1:\left(x_{n}^{\mathrm{SP}}-x_{n+1}^{\mathrm{SP}}\right)>l^{\mathrm{SP}}
\end{array}\right.
\end{gathered}
$$

where threshold value $l$ is set zero. Thus, increases between two successive BBI and SP, respectively are coded as " 1 " and consequently decreases and equilibrium are coded as " 0 ." Subsequently, $\mathbf{S}$ is subdivided into short sequences with a certain length. Each single word is obtained by a shift of one within the symbol string $\mathbf{S}$ [15]. The length of words is limited due to the requirement of a statistically sufficient representation of each single word type. To estimate the number of word types (histogram classes), the $\sqrt{N}$ approximation for histogram construction of $\mathrm{N}$ observations was used. For 30-min recordings (assumed mean heart rate: $80 \mathrm{bpm}$ ) there are no more than 64 different word types feasible. Taking into account the four different symbol combinations within $\mathbf{S}$ (the alphabets of BBI as well as SP consist each of two elements), words with a maximum length of three are realizable $\left(2^{3} * 2^{3}=64\right)$. Therefore, this approach is able to map the dynamics of BBI and SP within four consecutive heart beats (i.e. three BBI). From the perspective of phase space this corresponds to a three-dimensional embedding. Although this embedding is pragmatic rather then a true reconstruction of the system, several studies could show that a three-dimensional embedding of HRV is suitable to map the short-term dynamics [11], [16]. Moreover, the embedding dimension should not exceed $\log _{10}(N)$ to avoid spurious results [17]. Those mapped short-term fluctuations are 
predominantly influenced by respiratory activity and lead to high frequency (HF) oscillations in the power spectrum and the well-known phenomenon of respiratory sinus arrhythmia. To map longer-term dynamics a different approach is required (see Section II-C.).

In a first step, we consider the dynamics of BBI and SP within the word distribution density matrix $\mathbf{W}$ that contains the frequency of each of the $8 \times 8$ possible combinations of BBI and SP patterns (4)

$$
W=\left[\begin{array}{ccc}
\mathrm{BBI}_{000}, \mathrm{SP}_{000} & \cdots & \mathrm{BBI}_{000}, \mathrm{SP}_{111} \\
\vdots & \ddots & \vdots \\
\mathrm{BBI}_{111}, \mathrm{SP}_{000} & \cdots & \mathrm{BBI}_{111}, \mathrm{SP}_{111}
\end{array}\right]
$$

The Fuchs-Kennet-Outlier-Test (FKOT) (5) was applied to test whether the BBI and SP patterns of the particular word types occur independently and consequently to identify significantly present or absent word types.

Two observations $\mathrm{A}$ and $\mathrm{B}$ are independent, if $p(A) p(B)=p(A \wedge$ $B)$. Consequently, $p(A \wedge B)-p(A) p(B) \neq 0$ denotes dependency. In this context, the FKOT assesses the residuals between observed $\left(W_{j, k}\right)$ and expected $\left(E_{j, k}\right)$ word type frequencies [numerator of (5)], where $E_{j, k}=(1 / N) \sum_{m} W_{j, m} \sum_{n} W_{n, k}$ and $\mathrm{N}$ denotes the overall number of words [18].

To test whether those residuals are significant, normalization is performed (denominator of (5)) that takes the $(j-1)(k-1)$ degrees of freedom within $\mathbf{W}$ into account. (A frequently occurring word type results in a low frequency of the other word types in that row and column, respectively)

$$
u_{j, k}=\frac{W_{j, k}-E_{j, k}}{\sqrt{\frac{E_{j, k}}{N}\left(\left(1-\sum_{m} W_{j, m}-\sum_{n} W_{n, k}+E_{j, k}\right)\right.}} .
$$

Subsequently, the $u_{j, k}$ values are compared with standard normal distribution tables. Due to the $\mathrm{j} * \mathrm{k}$ multiple tests within $\mathbf{W}$ the significance level has to be corrected. Applying the correction proposed by Bonferoni [19] the corrected value $\mathrm{p}^{*}$ for a significance level of $\mathrm{p}=0.05$ is $\mathrm{p}^{*}=0.05 / 64=0.00078$.

Within $\mathbf{W}$ each word type was tested with FKOT whether its occurrence is below, within or above expectation. Using the standard normal distribution table values of $\mathrm{u}_{\mathrm{j}, \mathrm{k}}$ less than -3.37 reflect a significant under-representation and $u_{\mathrm{j}, \mathrm{k}}$ values above 3.37 reflect a significant over-representation of the specific word type.

\section{JSD Fractal Scaling Exponent}

To investigate the JSD of BBI and SP for long-term correlations, fractal scaling properties are assessed. Starting with the measured beat-to-beat-values stored in $\mathbf{X}$, a symbol transformation according to (6) is suggested

$$
s_{n}=\left\{\begin{aligned}
-1:\left(x_{n}^{\mathrm{BBI}}-x_{n+1}^{\mathrm{BBI}}\right)>l^{\mathrm{BBI}} \wedge\left(x_{n}^{\mathrm{SP}}-x_{n+1}^{\mathrm{SP}}\right)>l^{\mathrm{SP}} \\
1:\left(x_{n}^{\mathrm{BBI}}-x_{n+1}^{\mathrm{BBI}}\right)<l^{\mathrm{BBI}} \wedge\left(x_{n}^{\mathrm{SP}}-x_{n+1}^{\mathrm{SP}}\right)<l^{\mathrm{SP}} \\
0: \text { else }
\end{aligned}\right.
$$

where the threshold value $l$ is set to zero and $\wedge$ denotes logical "AND." Thus, in the symbolic vector $\mathbf{S}$ synchronous increase of BBI and SP are coded as "1," synchronous decreases of BBI and SP are coded as " -1 ," and all other characteristics as " 0 ." As the baroreflex (i.e. the blood pressure regulation via heart rate) is the predominant mechanism of short-term heart rate and blood pressure interactions the symbol coding focuses particularly on those patterns. Therefore, bradycardic baroreflex responses (i.e. a decrease of heart rate due to blood pressures increases) are coded as symbol " -1 " and tachycardic baroreflex responses (i.e. an increase of heart rate due to blood pressures drops) are coded as symbol " 1 ."

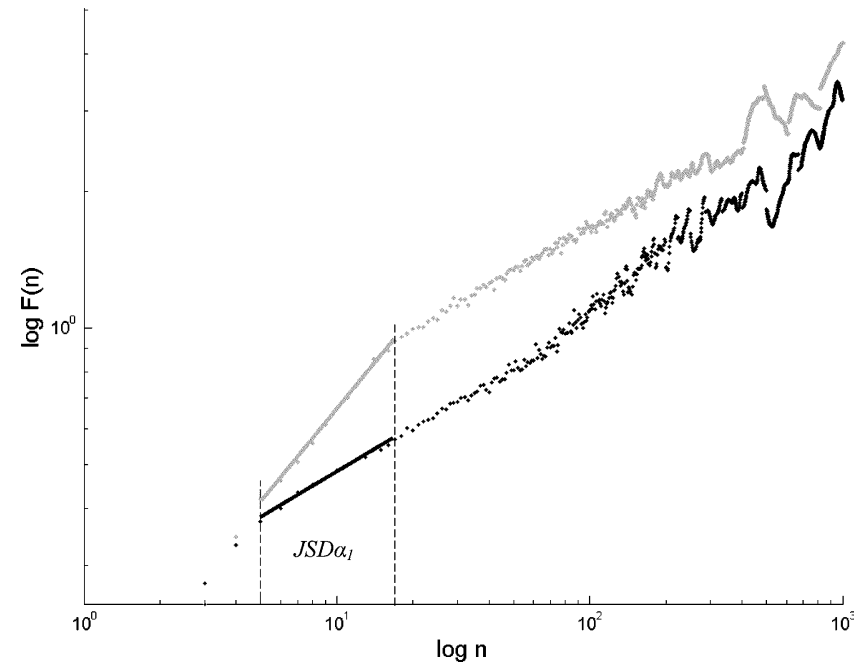

Fig. 1. Scaling properties of the symbol string derived from a healthy control (grey; $\mathbf{J S D} \boldsymbol{\alpha}_{1}=\mathbf{0 . 6 8}$ ) and Gaussian white noise (black; JSD $\boldsymbol{\alpha}_{1}=$ 0.33). $\boldsymbol{F}(\boldsymbol{n})$-root mean square of the detrended time series; $\boldsymbol{n}$-box size.

To investigate the presence of scaling invariance the detrended fluctuation analysis proposed by Peng et al. was applied [20]. The method works as follows.

1) Compute the cumulative sum $c(k)=\sum_{i=1}^{k}[s(k)-\bar{s}]$ of the symbol string $\mathbf{S}$ where $\bar{s}$ is the mean of $\mathbf{S}$ (using the concept of random-walk-analysis).

2) Compute the local trend $c_{n}(k)$ within boxes of varying sizes $n$ (least square fit).

3) Compute the root mean square of the detrended time series in dependency on box size $n$ as $F(n)=$ $\sqrt{(1 / N) \sum_{k=1}^{N}\left[c(k)-c_{n}(k)\right]^{2}}$, where $N$ denotes the size
of $\mathbf{S}$.

4) Plot $\log _{10} F(n)$ against $\log _{10} n$.

In the presence of scaling invariance, there is a linear relationship between $\log _{10} F(n)$ and $\log _{10} n$. As a measure of long-term correlation the scaling exponent $\alpha$ is computed (i.e. the slope of the line relating $\log _{10} F(n)$ to $\log _{10} n$ ) using least square fit (see Fig. 1). Values of $0<\alpha<0.5$ are associated with anti-correlation (i.e. large and small values of the time series are likely to alternate). For Gaussian white noise (i.e. random walk) $\alpha$ is 0.5 . Values of $0.5<\alpha \leq 1$ suggest the presence of power-law long-term correlations (i.e. large values of the time series are likely to be followed by large values) whereas $\alpha=1$ represents $1 / \mathrm{f}$ scaling. Values of $\alpha>1$ represent long-term correlations, but are different from the power-law.

For HRV analysis, two different regions of fractal scaling were found [20]. A characteristic break within the linear scaling graph was reported at a box size of $n=16$ and consequently suggested to compute two separate scaling exponents $\alpha_{1}$ for the range $n=4$ to $n=16$ and $\alpha_{2}$ for the range $n=16$ to $n=64$.

\section{Tests With Simulated Data}

To assess the impact of noise on the JSD, two independent Gaussian white noise processes were simulated for BBI and SP, respectively. To meet the 30-min recording length of the original data ten realizations were generated each consisting of 2000 values. The word distribution density matrix as well as the fractal scaling exponents were computed for all realizations and, thereafter, averaged.

\section{E. Sequence Method}

In a very simple model, the baroreflex is assumed to be a linear relationship between $\mathrm{SP}$ and $\mathrm{BBI}$ and the transfer factor is termed 
baroreflex sensitivity (BRS $=\Delta \mathrm{BBI} / \Delta \mathrm{SP}$ ). For baroreflex sensitivity estimation according to the sequence method, least square fit linear regression is performed between sequences $\mathrm{SP}_{i}$ of monotonic increasing blood pressure $\left(\mathrm{SP}_{i}=\left[\mathrm{SP}_{n}, \mathrm{SP}_{n+1}, \mathrm{SP}_{n+2}\right]\right.$ where $\mathrm{SP}_{n+2}>\mathrm{SP}_{n+1}>\mathrm{SP}_{n}$ ) that are followed by sequences $\mathrm{BBI}_{i}$ of monotonic increasing beat-to-beat intervals $\left(\mathrm{BBI}_{i}=\left[\mathrm{BBI}_{n+1}, \mathrm{BBI}_{n+2}, \mathrm{BBI}_{n+3}\right]\right.$ where $\mathrm{BBI}_{n+3}>\mathrm{BBI}_{n+2}>$ $\left.\mathrm{BBI}_{n+1}\right)$. The average of the regression line slopes $a_{i}(\mathrm{SP}, \mathrm{BBI})$ of all I sequences is then the BRS estimate (7)

$$
\mathrm{BRS}=\frac{1}{I} \sum_{i=1}^{I} a_{i}\left(\mathrm{SP}_{i}, \mathrm{BBI}_{i}\right) .
$$

Linear correlation coefficients $r_{x y}$ between BRS and JSD parameters were computed (8), where $x$ and $y$ denote the parameters and $\bar{x}$ and $\bar{y}$ their means, respectively

$$
r_{x y}=\frac{\sum_{i=1}^{n}\left(x_{i}-\bar{x}\right)\left(y_{i}-\bar{y}\right)}{\sqrt{\sum_{i=1}^{n}\left(x_{i}-\bar{x}\right)^{2} \sum_{i=1}^{n}\left(y_{i}-\bar{y}\right)^{2}}} .
$$

\section{RESULTS}

\section{A. Short-Term Joint Symbolic Dynamics (Word Distribution Matrix)}

The statistical analysis via FKOT showed that some word types within $\mathbf{W}$ are significantly over-represented and other ones are underrepresented. All word types representing symmetric BBI and SP behavior (i.e. $W_{1,1}, W_{2,2}, W_{3,3}, W_{4,4}, W_{5,5}, W_{6,6}, W_{7,7}$, and $W_{8,8}$ ) occur frequently, whereas some word types representing diametric behavior (i.e. $W_{7,2}, W_{5,4}, W_{4,5}$, and $W_{2,7}$ ) occur significantly seldom. Interpreting the former ones as baroreflex patterns and latter ones as missing or a lack of baroreflex responses the diagonals of the matrix were summed up: JSDsym $=(1 / N) \sum_{j=k=1}^{8} W_{j, k}$-representing symmetric word types and JSDdiam $=(1 / N) \sum_{j=k=1}^{8} W_{j, 9-k}$ representing diametric word types within $\mathbf{W}$.

In addition to the diagonals, there were some further significant word types. In both groups, word types $W_{5,1}, W_{7,5}, W_{8,7}$ and $W_{4,8}$ were over-represented and $W_{7,4}$ and $W_{2,5}$ were under-represented. Further, $W_{1,2}$ was over-represented and $W_{4,1}, W_{5,2}$ and $W_{8,2}$ were under-represented only in CON and $W_{2,4}$ was over-represented only in DCM. To consider the overall distribution the Shannon entropy within $\mathbf{W}$ was computed JSDshannon $=-\sum_{j, k=1}^{8}\left[\left(W_{j, k} / N\right) \log _{2}\left(W_{j, k} / N\right)\right]$. Comparing JSDsym, JSDdiam, and JSDshannon between CON and DCM there was a significant increase of JSDdiam in DCM.

\section{B. Long-Term Joint Symbolic Dynamics (Scaling Exponent)}

The analysis of scaling properties of the JSD showed a behavior similar to those of heart rate time series (see Fig. 1). The plot reveals scaling invariance in two regions. Therefore, two different scaling exponents were computed using the box sizes as proposed by Peng: JSD $\alpha_{1}$-scaling exponent for $n=4$ to $n=16$; JSD $\alpha_{2}$-scaling exponent for $n=16$ to $n=64$. In general, the slope in the small box size region $\left(\mathrm{JSD} \alpha_{1}\right)$ was higher than in the larger box size region (JSD $\left.\alpha_{2}\right)$. The comparison of DCM with CON (see Table I) showed significant differences in the scaling exponent $\operatorname{JSD} \alpha_{1}$ but not in $\mathrm{JSD} \alpha_{2}$.

\section{Gaussian White Noise}

Comparing the JSD parameters taken by the Gaussian white noise processes with those of the measured data of CON and DCM there were significances in JSDsym, JSDdiam, JSDshannon and JSD $\alpha_{1}$ (see Table I). Contrary, the JSD $\alpha_{2}$ exponents were all clustered together.
TABLE I

COMPARISON OF THE JSD PARAMETERS BETWEEN DCM PATIENTS, CONTROLS (CON) AND GAUSSIAN WHITE NOISE (GWN). TEST1-CON VERSUS DCM; TEST2-GWN VERSUS CON+DCM; SD-STANDARD DEVIaTION; SignifiCANCE LEVEL: $\mathbf{p}^{*}=\mathbf{0 . 0 1}$ (BONFERONI CORRECTED); N.S.-NOT SIGNIFICANT

\begin{tabular}{lcccccccc}
\hline & \multicolumn{2}{c}{ CON } & \multicolumn{2}{c}{ DCM } & \multicolumn{2}{c}{ GWN } & Test1 & Test2 \\
\hline parameter & mean & SD & mean & SD & Mean & SD & $\mathbf{p}^{*}$ & $\mathbf{p}^{*}$ \\
JSDsym & 0.34 & 0.14 & 0.31 & 0.15 & 0.15 & 0.01 & n.s. & $<0.001$ \\
JSDdiam & 0.04 & 0.03 & 0.05 & 0.05 & 0.16 & 0.01 & 0.006 & $<0.001$ \\
JSDshannon & 3.50 & 0.28 & 3.52 & 0.36 & 3.85 & 0.07 & n.s. & 0.001 \\
JSD $_{1}$ & 0.56 & 0.13 & 0.49 & 0.12 & 0.39 & 0.01 & 0.003 & 0.002 \\
JSD $_{2}$ & 0.33 & 0.05 & 0.35 & 0.07 & 0.33 & 0.02 & n.s. & n.s. \\
\hline
\end{tabular}

TABLE II

PEARSON'S LINEAR CORRELATION COEFFICIENTS BETWEEN JSD PARAMETERS AND BAROREFLEX SENSITIVITY. ( ${ }^{*}$-significance $<\mathbf{0 . 0 5}$; ** - significance $<0.01)$

\begin{tabular}{ll}
\cline { 2 - 2 } parameter & \multicolumn{1}{c}{ BRS } \\
\hline JSDsym & $0.26^{* *}$ \\
JSDdiam & $-0.25^{* *}$ \\
JSDshannon & -0.07 \\
JSD $\alpha_{1}$ & $0.19^{*}$ \\
\hline * - significance $<0.05 ; * *$ - significance $<0.01)$
\end{tabular}

\section{Correlation of JSD Parameters With Baroreflex Sensitivity}

The baroreflex sensitivity was significantly reduced in DCM compared with CON (CON: $12.3 \pm 7.9$ versus DCM: $6.3 \pm 4.9$; $\mathrm{p}<$ 0.001 ). The correlation analysis (see Table II) could not prove independence between JSD and BRS (except of JSDshannon). However, the linear correlations between JSD parameters and BRS were weak.

\section{DISCUSSION}

The analysis of the JSD of BBI and SP by means of a word distribution matrix showed the presence of deterministic components as well as correlations between BBI and SP. Applying the Fuchs-KennetOutlier-Test we concluded that this approach is able to quantify dynamics underlying the heart rate and blood pressure regulation. Although this test was originally designed for independent samples it also provides the detection of statistically over- and under-represented word types. The word distribution matrix in DCM revealed a significantly increased number of diametric word types in comparison with that of CON. Since those word types map in an abstract manner a behavior oppositional to the typical baroreflex response, this finding might be partly interpreted as a loss of baroreflex mediated regulation in DCM. Furthermore, the number of frequent or seldom word types was less in DCM than in CON. This also points at a loss of HRV mediated by an impaired blood pressure regulation in DCM that has been described by other authors [21].

The analysis of the JSD scaling properties showed that the proposed symbol coding maps joint heart rate and blood pressure dynamics up to box sizes of 16 heart beats since the JSD $\alpha_{1}$ values of measured BBI and SP data were far from of those of Gaussian white noise. JSD $\alpha_{1}$ of Gaussian noise is considerably less than 0.5 (i.e. random walk) due to the symbol coding where symbol ' 0 ' is more likely than symbol " 1 " and " -1 ," respectively. In $\mathrm{CON}, \mathrm{JSD} \alpha_{1}$ was slightly above 0.5 and, therefore, suggests the presents of long-term correlations. Considering that the symbol coding focused only on baroreflex response-like patterns, this finding is in accordance with the understanding of the underlying physiology. The sympathetically mediated baroreflex has 
duration of about ten seconds [22] and would result in a long-term correlation within the symbol string that is covered by the box sizes of JSD $\alpha_{1}$. Contrary, JSD $\alpha_{1}$ was less than 0.5 in DCM. This finding might partly result from alternans phenomena that were already observed in heart rate and blood pressure time series of DCM patients [23]. JSD $\alpha_{2}$ does not reflect baroreflex-like heart rate and blood pressure interactions and seems to be mainly affected by noise as the values are clustered with those of the simulated Gaussian process. Possibly, higher threshold values for the symbol coding might lead to a better distinction of scaling phenomena and should, therefore, be a subject of future investigations.

The weak correlations between JSD parameters and BRS computed with the sequence method (i.e. the standard measure for heart rate and blood pressure interaction analysis) on one hand, and the significant differences of the diametric word types (short-term) and $\alpha_{1}$-scaling exponents (long-term) between CON and DCM on the other hand, endorse the importance of supplementary methods for interaction analyses and, furthermore, recommend the approach proposed here. In an advantage over standard methods, short- and long-term JSD also consider nonlinear interactions and furthermore provide easy-to-interpret physiological patterns. Therefore, this might be a useful tool for enhanced analyses of DCM as well as other cardiovascular diseases.

\section{REFERENCES}

[1] F. Lombardi, "Clinical implications of present physiological understanding of HRV components," Card. Electrophysiol. Rev., vol. 6, no. 3, pp. 245-249, Sep. 2002.

[2] P. A. Lanfranchi and V. K. Somers, "Arterial baroreflex function and cardiovascular variability: interactions and implications," Am. J. Physiol. Regul. Integr. Comp. Physiol., vol. 283, no. 4, pp. R815-R826, Oct. 2002.

[3] V. Eijzenbach, J. H. Sneek, and C. Borst, "Arterial pressure and heart period in the conscious rabbit: diurnal rhythm and influence of activity," Clin. Exp. Pharmacol. Physiol., vol. 13, no. 8, pp. 585-592, Aug. 1986.

[4] S. Akselrod, S. Eliash, S. Oz, and O. Cohen, "Hemodynamic regulation in SHR: investigation by spectral analysis," Am. J. Physiol., pt. 2, vol. 253, no. 1, pp. H176-H183, Jul. 1987.

[5] G. Baselli, E. Caiani, A. Porta, N. Montano, M. G. Signorini, and S. Cerutti, "Biomedical signal processing and modeling in cardiovascular systems," Crit. Rev. Biomed. Eng., vol. 30, no. 1-3, pp. 55-84, 2002.

[6] G. Bertinieri, M. di Rienzo, A. Cavallazzi, A. U. Ferrari, A. Pedotti, and G. Mancia, "A new approach to analysis of the arterial baroreflex," $J$. Hyperten. Suppl., vol. 3, no. Suppl 3, pp. 79-81, Dec. 1985.

[7] M. A. Cohen and J. A. Taylor, "Short-term cardiovascular oscillations in man: measuring and modeling the physiologies," J. Physiol., pt. 3, vol. 542, pp. 669-683, Aug. 1, 2002.

[8] B. Pompe, P. Blidh, D. Hoyer, and M. Eiselt, "Using mutual information to measure coupling in the cardiorespiratory system," IEEE Eng. Med. Biol. Mag., vol. 17, no. 6, pp. 32-39, Nov.-Dec. 1998.

[9] A. Porta, G. Baselli, F. Lombardi, N. Montano, A. Malliani, and S. Cerutti, "Conditional entropy approach for the evaluation of the coupling strength," Biol. Cybern., vol. 81, no. 2, pp. 119-129, Aug. 1999.

[10] J. Hadamard, "Les surfaces a courbures opposees et leurslignes geodesiques," J Math. Pure Appl., 1898.

[11] A. Voss, J. Kurths, H. J. Kleiner, A. Witt, N. Wessel, P. Saparin, K. J. Osterziel, R. Schurath, and R. Dietz, "The application of methods of nonlinear dynamics for the improved and predictive recognition of patients threatened by sudden cardiac death," Cardiovasc. Res., vol. 31, no. 3, pp. 419-433, Mar. 1996.

[12] A. C. Yang, S. S. Hseu, H. W. Yien, A. L. Goldberger, and C. K. Peng, "Linguistic analysis of the human heartbeat using frequency and rank order statistics," Phys. Rev. Lett., vol. 90, no. 10, Mar. 14, 2003.

[13] H. Malberg, N. Wessel, A. Hasart, K. J. Osterziel, and A. Voss, "Advanced analysis of spontaneous baroreflex sensitivity, blood pressure and heart rate variability in patients with dilated cardiomyopathy," Clin. Sci. (Lond.), vol. 102, no. 4, pp. 465-473, Apr. 2002.

[14] N. Wessel, A. Voss, H. Malberg, C. Ziehmann, H. U. Voss, A. Schirdewan, U. Meyerfeldt, and J. Kurths, "Non-linear analysis of complex phenomena in cardiological data," Herzschrittmachertherapie und Elektrophysiologie, vol. 11, no. 3, pp. 159-173, 2000.
[15] M. Baumert, T. Walther, J. Hopfe, H. Stepan, R. Faber, and A. Voss, "joint symbolic dynamic analysis of beat-to-beat interactions of heart rate and systolic blood pressure in normal pregnancy," Med. Biol. Eng. Comput., vol. 40, no. 2, pp. 241-245, 2002.

[16] M. Baumert, V. Baier, J. Haueisen, N. Wessel, U. Meyerfeldt, A. Schirdewan, and A. Voss, "Forecasting of life threatening arrhythmias using the compression entropy of heart rate," Meth. Inf Med., vol. 43, no. 2, pp. 202-206, 2004.

[17] A. M. Albano, A. I. Mees, G. C. de Guzman, and P. E. Rapp, "Data requirements for reliable estimation of correlation dimensions," in Chaos in Biological Systems, H. Degn, A. V. Holden, and L. F. Olsen, Eds. New York: Plenum, 1987, pp. 207-220.

[18] C. Fuchs and R. Kennet, "A test for detecting outlying cells in the multinormal distribution and two-way contingency tables," J. Am. Statist. Assoc., vol. 75, pp. 395-398, 1980.

[19] G. E. P. Box, W. G. Hunter, and J. S. Hunter, Statistics for Experimenters. New York: Wiley, 1978.

[20] C. K. Peng, S. Havlin, H. E. Stanley, and A. L. Goldberger, "Quantification of scaling exponents and crossover phenomena in nonstationary heartbeat time series," Chaos, vol. 5, no. 1, pp. 82-87, 1995.

[21] C. Rostagno, M. Felici, S. Caciolli, G. Olivo, M. Comeglio, G. Galanti, and G. G. Serneri, "Decreased baroreflex sensitivity assessed from phase IV of Valsalva maneuver in mild congestive heart failure," Angiology, vol. 50, no. 8, pp. 655-664, Aug. 1999.

[22] R. W. DeBoer, J. M. Karemaker, and J. Strackee, "Hemodynamic fluctuations and baroreflex sensitivity in humans: a beat-to-beat-model," $\mathrm{Am}$. J. Physiol., pt. 2, vol. 7, no. 3, pp. H680-H689, 1998.

[23] U. Leder, H. P. Pohl, V. Baier, M. Baumert, M. Liehr, J. Haueisen, A. Voss, and H. R. Figulla, "Alternans of heart rate and blood pressure in dilated cardiomyopathy," PACE, vol. 25, no. 9, pp. 1307-1314, 2002.

\section{Decomposition of Three-Dimensional Medical Images Into Visual Patterns}

Raquel Dosil*, Xosé M. Pardo, and Xosé R. Fdez-Vidal

Abstract-In this paper, we present a method for the decomposition of a volumetric image into its most relevant visual patterns, which we define as features associated to local energy maxima of the image. The method involves the clustering of a set of predefined bandpass energy filters according to their ability to segregate the different features in the image, thus generating a set of composite-feature detectors tuned to the specific visual patterns present in the data. Clustering is based on a measure of statistical dependence between pairs of frequency features. We will illustrate the applicability of the method to the initialization of a three-dimensional geodesic active model.

Index Terms-Active model initialization, low level representation, multiresolution analysis, phase congruence.

\section{OBJECTIVES}

In this paper, we present a method for low-level representation of three-dimensional (3-D) images consisting on the identification its most relevant low-level features, which we call visual patterns. Some

Manuscript received July 19, 2004; revised April 17, 2005. This work was supported in part by the Xunta de Galicia under Grant PGIDIT04TIC206005PR. Asterisk indicates corresponding author.

*R. Dosil is with the Department of Electronics and Computer Science, Universidade de Santiago de Compostela. Campus Universitario Sur, s/n, 15782 Santiago de Compostela, Spain (e-mail: rdosil@usc.es).

X. M. Pardo the Department of Electronics and Computer Science, Universidad de Santiago de Compostela, 15782 Santiago de Compostela, Spain.

X. R. Fdez-Vidal is with Department of Applied Physics, Universidade de Santiago de Compostela. Escola Politécnica Superior, 27002 Lugo, Spain.

Digital Object Identifier 10.1109/TBME.2005.857635 